\title{
Substructure and Tidal Streams in the Andromeda Galaxy and its Satellites
}

\author{
Annette M. N. Ferguson and A. D. Mackey
}

\begin{abstract}
Tidal streams from existing and destroyed satellite galaxies populate the outer regions of the Andromeda galaxy (M31). This inhomogeneous debris can be studied without many of the obstacles that plague Milky Way research. We review the history of tidal stream research in M31, and in its main satellite galaxies. We highlight the numerous tidal streams observed around M31, some of which reside at projected distances of up to $\sim 120 \mathrm{kpc}$ from the center of this galaxy. Most notable is the Giant Stellar Stream, a signature of the most recent significant accretion event in the M31 system. This event involved an early-type progenitor of mass $\sim 10^{9} \mathrm{M}_{\odot}$ that came within a few kpc of M31's center roughly a gigayear ago; almost all of the inner halo $(\mathrm{R} \leq 50 \mathrm{kpc})$ debris in $\mathrm{M} 31$ can be tied either directly or indirectly to this event. We draw attention to the fact that most of M31's outer halo globular clusters lie preferentially on tidal streams and discuss the potential this offers to use these systems as probes of the accretion history. Tidal features observed around M33, M32, NGC 205 and NGC 147 are also reviewed. We conclude by discussing future prospects for this field.
\end{abstract}

\section{Introduction}

Within the context of the cold dark matter paradigm, structure formation proceeds hierarchically and galaxies like the Milky Way and Andromeda (M31) are predicted to arise from the merger and accretion of many smaller sub-systems as well as from

A. M. N. Ferguson

Institute for Astronomy, University of Edinburgh, Blackford Hill, Edinburgh EH9 3HJ, UK

e-mail: fergusoneroe.ac.uk

A. D. Mackey

Research School of Astronomy \& Astrophysics, Australian National University, Mount Stromlo Observatory, Cotter Road, Weston Creek, ACT 2611, Australia

e-mail: dougal.mackeyeanu.edu.au 
the smooth accretion of intergalactic gas (e.g White \& Rees, 1978; White \& Frenk, 1991). Galaxy outskirts are of particular interest since the long dynamical timescales in these regions mean that coherent debris from past accretion events has the greatest longevity. The discovery of the Sagittarius dwarf galaxy and associated tidal stream (see Law \& Majewski, this volume) demonstrated beyond a doubt that satellite accretion played an important role in the growth of the Milky Way's halo, but the veracity of this aspect of the hierarchical model could not rest solely on a single event observed within a single galaxy. In this spirit, the late 90s saw the quest begin to identify other galactic systems which were visibly in the process of devouring smaller satellites.

Our nearest giant neighbour, M31, provides the most obvious target for such studies. Lying at $\sim 780 \mathrm{kpc}$, it is in many respects a sister galaxy to the Milky Way. It has a similar total mass (e.g. Diaz et al., 2014; Veljanoski et al., 2014), is of a similar morphological type, and it resides in the same low-density environment one which is deemed typical for much of the present-day galaxy population. M31's proximity means that individual stars near the tip of the red giant branch (RGB) can be resolved from the ground; this offers a powerful method for probing very low effective surface brightnesses, such as those expected for tidal debris streams. Furthermore, its high inclination to the line-of-sight $\left(i \sim 77^{\circ}\right)$ means that it is ideally suited for studies of its halo regions.

There are some disadvantages to studying tidal streams in M31 as opposed to our own Milky Way. Even with the world's largest telescopes, ground-based studies of M31's halo are limited to using luminous giant stars whereas Milky Way studies can harness the power of the much more numerous main sequence turn-off population (e.g. Belokurov et al., 2006). Additionally, in M31 we are mainly confined to analysis of projected positions on the sky (although occasionally some line-of-sight distance information is available), and we can only measure radial velocities. This can be contrasted with the situation in the Milky Way where it is possible to additionally determine line-of-sight distances 1 and proper motions, and thus probe the full six dimensional phase space. In effect, these differences imply that in M31 we are sensitive to tidal streams that are of higher surface brightness than those we can uncover in the Milky Way, and moreover typically only the subset retaining a high degree of spatial and/or kinematical coherence.

On the other hand, there are some clear advantages to studying an external system such as M31. Our vantage point largely alleviates complicated line-of-sight projection and extinction effects, such as must be endured in studies of the Milky Way. This means that we have a better understanding of the morphology of tidal features and where stellar substructures lie (at least in projection) with respect to each other, and with respect to additional halo tracers such as globular clusters (GCs) and dwarf satellites. Our bird's-eye view also makes it fairly straightforward to construct in situ samples of halo stars at various radii. Remarkably, we currently know more about the outer halo $(\mathrm{R} \gtrsim 50 \mathrm{kpc}$ ) of M31 than we do of the Milky Way. In the Milky Way,

\footnotetext{
${ }^{1}$ In practice, individual distances to large samples of stars in the Milky Way are crude at the moment. ESA's Gaia mission will change this when it starts to deliver data in 2016 but the most accurate distances will be limited to stars within roughly $10 \mathrm{kpc}$ of the Sun.
} 
the outer halo is obscured by a dense veil of foreground stars making the robust identification of the low density population of outer halo stars difficult. In addition, while the main sequence turn-off method so extensively used by the Sloan Digital Sky Survey (SDSS) teams has probed out to distances of $\sim 40 \mathrm{kpc}$, such stars are not detected in SDSS imaging at distances beyond this. While some outer halo substructures have been uncovered using other tracers (e.g. Newberg et al., 2003; Watkins et al., 2009), the inhomogeneous and sparse nature of these studies precludes any meaningful conclusions about the global properties of the Milky Way's outer halo. In M31, we also have a far clearer view of the low latitude regions of the galaxy, enabling discrimination between perturbed disk features and accreted substructures.

Over the last 15 years, a multitude of studies have targeted the outskirts of M31; this chapter reviews the tremendous progress and exciting results that have emerged from this work.

\section{Historical Studies}

While the first detailed studies of faint structure and substructure in M31 did not appear until the early 2000s, there were a number of studies prior to this which are of particular note.

Baade \& Gaposchkin (1963) were the first to comment on the strong warp present in the outer parts of M31's stellar disk. Baade (p. 73) notes that the disk signature is still present at a major axis distance of $2^{\circ}$ but that the opposing sides of the disk have "swirled" off in anti-symmetric directions by a radius of $2.25^{\circ}$ - an observation he speculates could be due to the tidal action of the Milky Way on M31. The existence of the prominent stellar warp was further confirmed by Innanen et al. (1982) who stacked digital Palomar Schmidt plates to reach surface brightnesses in M31 of $\mu_{\mathrm{V}} \gtrsim 25.8 \mathrm{mag} \operatorname{arcsec}^{-2}$. Early HI studies of M31 also revealed a strong warp in the neutral hydrogen disk (e.g. Roberts \& Whitehurst, 1975; Newton \& Emerson, 1977; Cram et al., 1980). While the stellar and HI disks are warped in the same direction, the stellar warp appears to begin at a smaller radius than the H I warp and it exhibits a greater deviation from the disk plane; this holds true along both major axes but seems especially apparent in the north-east.

Walterbos \& Kennicutt (1988) conducted the definitive study of the light distribution in M31 before the era of wide-field resolved star mapping, including a first quantitative exploration of the peripheral disk. They constructed multi-band images from digitised Burrell Schmidt plates and analysed surface brightness and color profiles across the disk. They also detected a clear warp, but speculated that the north-eastern warp, due to its faintness and extreme bend, may actually be a galactic reflection nebula and not a stellar feature associated with M31. This issue was settled by Morris et al. (1994) who resolved the stellar populations in the north-eastern warp (which they termed the 'Spur') for the first time and showed they lay at the distance of M31. Fig. 1 of Walterbos \& Kennicutt (1988) shows beyond doubt that 
the north-eastern half of the disk is far more perturbed than the south-western half, and, in hindsight, one can even see a slight luminosity enhancement in the direction of the Giant Stellar Stream.

Equally influential were the first studies of M31's resolved stellar halo. Mould \& Kristian (1986), Crotts (1986) and Pritchet \& van den Bergh (1988) all presented colourmagnitude diagrams (CMDs) of small regions located at several kpc along M31's minor axis, a region perceived at the time to be dominated by pure halo. These studies revealed a moderate metallicity population $([\mathrm{Fe} / \mathrm{H}] \sim-1)$ with a large metallicity spread, in stark contrast to the metal-poor halo of the Milky Way. The disparity of the halo populations in two galaxies that were otherwise considered rather similar remained a significant puzzle throughout the following decade.

\section{Wide-Field Mapping Surveys of M31}

The relative proximity of M31, while advantageous for detailed study, also poses a problem in that even the main body of the galaxy subtends a substantial angle on the sky. In the mid-to-late 90s, wide-format CCD detectors became increasingly available on medium-sized telescopes and this development opened up new possibilities for surveying the faint outlying regions of M31. With a distance modulus of $m-M=24.47 \pm 0.07$ (McConnachie et al., 2005), stars near the tip of M31's RGB $\left(M_{I} \approx-4\right)$ have $I \approx 20.5$ and thus could be easily detected in modest exposures with a 2-m class telescope. As a result, it became feasible to contiguously map resolved stars at the bright end of the luminosity function over very large areas.

RGB stars are the evolved counterparts of low to intermediate mass $(\mathrm{M} \sim 0.3-8$ $\mathrm{M}_{\odot}$ ) main-sequence stars with ages of at least $\gtrsim 1 \mathrm{Gyr}$. While asymptotic giant branch stars and high mass main sequence stars can be even more luminous than RGB stars, RGB stars are the most interesting in the context of tidal stream research since they are long-lived and can be used to trace the old stellar components of galaxies, where signatures of hierarchical assembly are expected to be most prevalent. Additionally, for a fixed age, the colour of an RGB star depends almost entirely on its metallicity; thus, for a roughly uniform age for a stellar population, individual stellar metallicities can be derived purely from photometry.

Resolved star surveys map the spatial distribution of individual RGB stars which can in turn be used to infer the surface brightness distribution of the underlying light. This technique allows much lower surface brightness levels to be reached than typically achievable with conventional analyses of diffuse light. As a simplistic illustration of how the method works, consider a population of M31 RGB tip (TRGB) stars with $I_{0} \sim 20.5$ and $(V-I)_{0} \sim 1.5$. A surface density of $10^{5}$ such stars per square degree corresponds to $\mu_{V} \approx 27$ mag $\operatorname{arcsec}^{-2}$ while a surface density of $10^{3}$ stars per square degree corresponds to $\mu_{V} \approx 32 \mathrm{mag} \operatorname{arcsec}^{-2}$. This calculation is crude since it neglects the fact that there is a range of RGB luminosities within a population, and also that some sizeable fraction of the total light will come from stars fainter than the magnitude limit, but these are corrections that can be easily calculated for 
any given survey (e.g. Pritchet \& van den Bergh, 1994; McConnachie et al., 2010). Nonetheless, it is sufficient to demonstrate that, in the low crowding outer regions of M31 (and external galaxies in general), the resolved RGB star technique is clearly the optimal means to search for and map very faint structures. The main challenges in using this method are to image to sufficient depth to detect a statistically significant population of RGB halo stars, and to disentangle genuine RGB stars from contaminating populations, which typically consist of foreground Milky Way dwarf stars and unresolved background galaxies (see the left panel of Fig.11).

The breakthrough in our ability to search for low surface brightness structure in the outskirts of M31 led to the discovery of a plethora of faint streams and substructure in the the inner halo, including the dominant Giant Stellar Stream. The pioneering INT Wide Field Camera survey (e.g. Ibata et al., 2001; Ferguson et al., 2002; Irwin et al., 2005) mapped $\approx 40$ square degrees ( 163 contiguous pointings) around M31 in the $V$ and $i$ passbands, reaching to $\sim 3$ magnitudes below the TRGB (see the right panel of Fig. 11). M31 was also targeted by the SDSS (Zucker et al., 2004a), where stars near the TRGB were mapped to large distances along the major axis. These surveys also uncovered several previously-unknown M31 dwarf satellites and globular clusters (e.g. Zucker et al., 2004b, 2007; Irwin et al., 2008; Huxor et al., 2008).

In parallel to these efforts to survey RGB stars, other groups began to explore the halo and outer disk populations using planetary nebulae (PNe) (e.g. Merrett et al, 2003, 2006; Morrison et al., 2003; Kniazev et al., 2014). Although PNe offer some advantages over RGB stars as tracers of halo light (for example, they are more luminous, suffer much reduced sample contamination, and can provide simultaneous information on radial velocities), they are far rarer. From a survey of PNe in the M31 bulge, Ciardullo et al. (1989) found that the ratio between the number of PNe in the top 2.5 magnitudes of the luminosity function and the $V$-band luminosity was $\alpha_{2.5} \sim 30.8 \times 10^{-9} \mathrm{PNe}$ per $\mathrm{L}_{\odot}$. This leads to the expectation of $\approx 50$ PNe per square degree at $\mu_{V} \sim 24 \mathrm{mag} \operatorname{arcsec}^{-2}$ but only $\approx 1$ per square degree at $\mu_{V} \sim 28$ mag $\operatorname{arcsec}^{-2}$. This makes surveys for resolved RGB stars far more efficient than those for PNe in the outer regions of M31. However, the situation changes for more distant galaxies, where RGB stars become too faint to resolve while PNe can still be detected (e.g. Coccato et al., 2013; Foster et al., 2014).

Based on the success of these early studies, exploration of the M31 outer halo began in the mid 2000s. Due to the lower stellar density in these parts, deeper photometry was required in order to sufficiently sample the RGB luminosity function and this in turn required larger telescopes. The Pan-Andromeda Archaeological Survey (PAndAS) was conducted using the MegaCam instrument on the 3.8-m CFHT to contiguously map over 380 square degrees around the M31-M33 region and detect stars to $\sim 4$ mag below the TRGB (e.g. Ibata et al., 2007; McConnachie et al., 2009: Ibata et al., 2014). Other wide-field ground-based work concentrated on deep pencil beam studies of the outer halo (e.g. Ostheimer, 2003; ;anaka et al., 2010). 

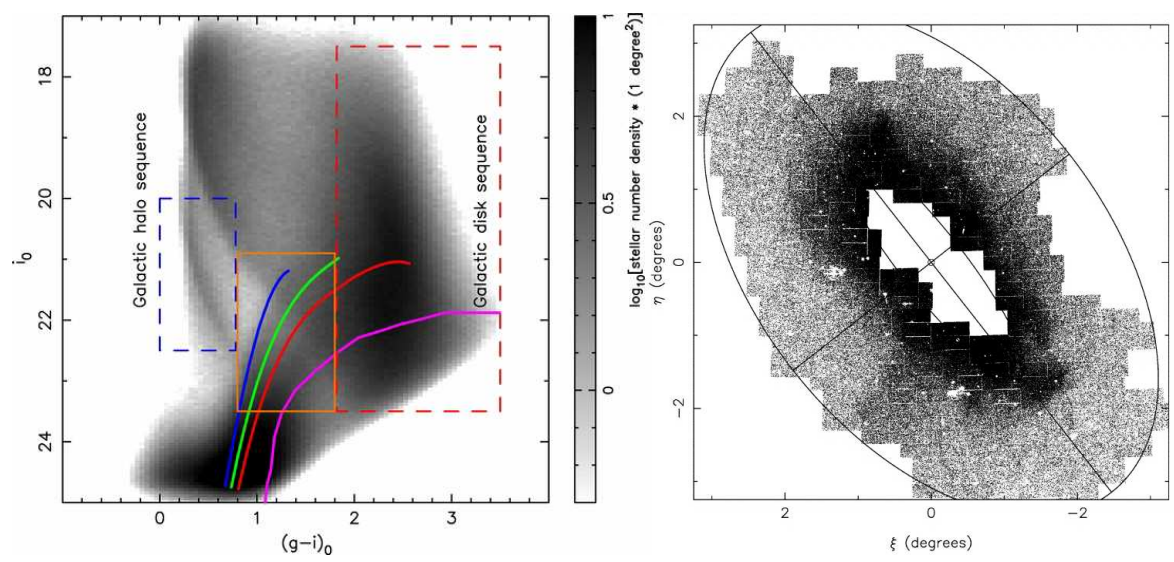

Fig. 1: (Left) A Hess diagram of the point sources in the PAndAS survey at distances beyond $2^{\circ}$ of M31 (reproduced from Ibata et al., 2014) . A series of fiducial tracks spanning $[\mathrm{Fe} / \mathrm{H}]=-1.91,-1.29,-0.71$ and -0.2 are superimposed on the RGB while contaminating Milky Way foreground disk and halo sequences are indicated by dashed boxes. Blueward of $(g-i)_{0} \sim 1$ and fainter than $i_{0} \sim 23$, unresolved background galaxies become the primary contaminant. The orange box shows the adopted colour-magnitude selection for M31 RGB stars. (Right) An early map of metal-poor RGB star counts around M31 from the INT/WFC survey (reproduced from Ferguson et al., 2002). The outer ellipse has a flattening of 0.6 and a semimajor axis length of $55 \mathrm{kpc}$.

\section{Major Tidal Features in the Halo of M31}

Figs. 2 and 3 show maps from the PAndAS survey of RGB stars in the inner and outer halo regions of M31, displayed to highlight prominent substructures. Additionally, Fig. 4 4 shows the distribution of RGB stars in four different metallicity bins, revealing how the morphology of the tidal debris changes as a function of metallicity. A striking feature of all of these visualisations is the non-uniformity of the stellar distribution in the outer regions. The most metal-poor map presented in Fig. 4has a smoother appearance than the others, but it still exhibits a substantial degree of substructure.

The inner halo ( $\mathrm{R}<50 \mathrm{kpc}$ ) of M31 appears as a flattened structure (axis ratio $\sim 0.5$ ) in Fig. 2, around the edge of which bright tidal features (e.g. streams, clumps, spurs, shelves) can be seen. In the outer halo $(\mathrm{R} \geq 50 \mathrm{kpc})$, the most prominent features are a multitude of faint narrow streams and arcs. Based on their appearance in Fig. 4, these outer streams are also considerably more metal-poor than the substructures which dominate the inner halo. Lewis et al. (2013) searched for a correlation between the tidal structures seen in stars, and features in the HI gas around M31. 
Interestingly, they found a general lack of spatial correlation between these two components on all scales, with very few potential overlaps.

A brief description of some of the most prominent tidal features seen around M31 is given below:

- Giant Stellar Stream (GSS): Discovered in the first quadrant of M31 that was mapped by the INT/WFC survey, the GSS is the most prominent overdensity in M31's halo and covers a large fraction of its south-east quadrant (Ibata et al., 2001). It can be traced as a coherent structure to a projected galactocentric radius of $\sim 100 \mathrm{kpc}$, and spans a width of $\sim 25 \mathrm{kpc}$. The stream has a linear morphology with a sharp eastern edge and an estimated absolute $V$-band magnitude of $M_{V} \approx-14$ (Ibata et al, 2001). However, this is a crude estimate based on only that part of the stream which is visible in the earliest INT maps. As the stream is now known to be more than twice as long as this, and other debris features have been identified as forward wraps of the structure, the total luminosity of the GSS could easily be 1-2 magnitudes higher. Ibata et al. (2007) show that there is a large-scale stellar population gradient present, with the high surface brightness core region of the stream having relatively more metal-rich stars than the peripheral regions. Both photometric and spectroscopic studies reveal the core stream to have a moderately high metallicity of $[\mathrm{Fe} / \mathrm{H}] \geq-0.5$ to -0.7 , with the envelope dropping to $[\mathrm{Fe} / \mathrm{H}] \sim-1.4$ (Guhathakurta et al., 2006; Ibata et al., 2007; Gilbert et al., 2009).

- G1 Clump: This feature was first recognised in the INT map published by Ferguson et al. (2002) and appears as a rather round clump of stars located at a projected radius of $\sim 30 \mathrm{kpc}$ along the south-western major axis of M31. It has dimensions of $0.5^{\circ} \times 0.7^{\circ}$, or $7 \times 10 \mathrm{kpc}$ at the distance of M31. Ferguson et al. (2002) estimate an absolute magnitude of $M_{V} \approx-12.6$ and a $V$-band surface brightness of $\approx 28.5 \mathrm{mag} \operatorname{arcsec}^{-2}$. The feature was originally named because the luminous M31 globular cluster G1 lies nearby. This star cluster is notable because it has been argued to have both an internal metallicity spread as well as an intermediate-mass black hole, characteristics that suggest it could be the remnant core of a nucleated dwarf elliptical galaxy (e.g. Meylan et al., 2001; Gebhardt et al., 2005). While the detection of tidal debris in the vicinity of this enigmatic object was very exciting, subsequent observations of the properties of stars in the G1 Clump appear to rule out any association between the two (Rich et al., 2004; Reitzel et al., 2004; Ibata et al., 2005; Faria et al., 2007).

- North-East Clump (NE Clump): Located at a projected radius of $\sim 40 \mathrm{kpc}$ and near the north-eastern major axis, this substructure is one of the most nebulous features in the inner halo of M31. It subtends a diameter of $\sim 1^{\circ}$ or $\sim 14 \mathrm{kpc}$ at the distance of M31, and appears to connect to the main body of the galaxy by a faint filament. It is estimated to have an absolute $g$-band magnitude of $M_{g} \approx-11.6$ and a $g$-band surface brightness of $\approx 29.0 \mathrm{mag} \mathrm{arcsec}^{-2}$ (Zucker et al., 2004a). Although it was initially suggested that the NE Clump was a disrupting dwarf satellite, subsequent observations have disfavoured this interpretation (Ibata et al., 2005; Richardson et al., 2008; Bernard et al., 2015a), since the stellar populations are more representative of the disk. 
- North-Eastern (NE) and Western (W) Shelves: The NE Shelf is a diffuse but fairly sharp-edged extension lying north-east of M31's center, while the Western Shelf is a fainter feature of similar morphology and size on the opposite side of the galaxy. On the basis of their comparably high metallicities, Ferguson et al. (2002) suggested the NE Shelf could be an extension of the GSS. Using inferences from $N$-body simulations, Fardal et al. (2007) argued that both the NE and $\mathrm{W}$ Shelves were forward continuations of the stream, representing material stripped off during successive pericentric passages.

- Streams B, C, D: Identified by Ibata et al. (2007), Streams B-D are a series of approximately parallel tangential streams which cross the southern minor axis of M31 inside a radius of $100 \mathrm{kpc}$. Their metallicities are in the range $-1.5 \leq[\mathrm{Fe} / \mathrm{H}] \leq-0.5$. The eastern portions of Streams $\mathrm{C}$ and $\mathrm{D}$ appear to overlap in projection and all of the streams seem to terminate, or at least dramatically fade, once they reach the GSS. Stream C has been shown to be particularly complex, consisting of two distinct (but overlapping) metallicity and kinematic components (Chapman et al., 2008; Gilbert et al., 2009). Along with the GSS, the metal-rich component of Stream C is the only other outer halo feature visible in the most metal-rich maps of Fig. 4

- Far Outer Halo Streams $(\mathbf{R} \gtrsim 100 \mathbf{~ k p c})$ : Figs. 3 and 4 show that the outer halo is littered with various faint streams and clumps, including Stream A, the North-West (NW) Stream, the South-West (SW) Cloud))) and the (((Eastern (E) Cloud (Ibata et al., 2007; McConnachie et al., 2009; Richardson et al., 2011; Carlberg et al., 2011; Bate et al., 2014). With $\mu_{\mathrm{V}} \geq 31.5 \mathrm{mag} \operatorname{arcsec}^{-2}$, these streams represent the faintest spatially-coherent debris yet identified around M31. Stream A crosses the minor axis at a projected radius of $\sim 120 \mathrm{kpc}$ and aligns with the inner network of tangential streams. The E and SW Clouds appear as stellar arcs at slightly smaller radii on either side of Stream A. The NW Stream is a long $(\sim 100 \mathrm{kpc})$ and narrow $(\sim 3 \mathrm{kpc})$ radial feature; although there is no visible connection between the upper and lower branches of the stream, the fact that both trace out segments of a single ellipse and have a similar metallicity has supported the notion they are related (Carlberg et al., 2011). Although part of the NW Stream was also seen in the deep pencil beam study of Tanaka et al. (2010) (their (Stream F), their Stream E was not recovered in the PAndAS survey.

\section{Understanding the Nature and Origin of Tidal Features in M31}

Following the initial discovery of tidal features in the M31 halo, concerted followup observations and detailed modelling have been carried out in order to develop a complete understanding of this material. In this section, we review and summarise some highlights from this work. 


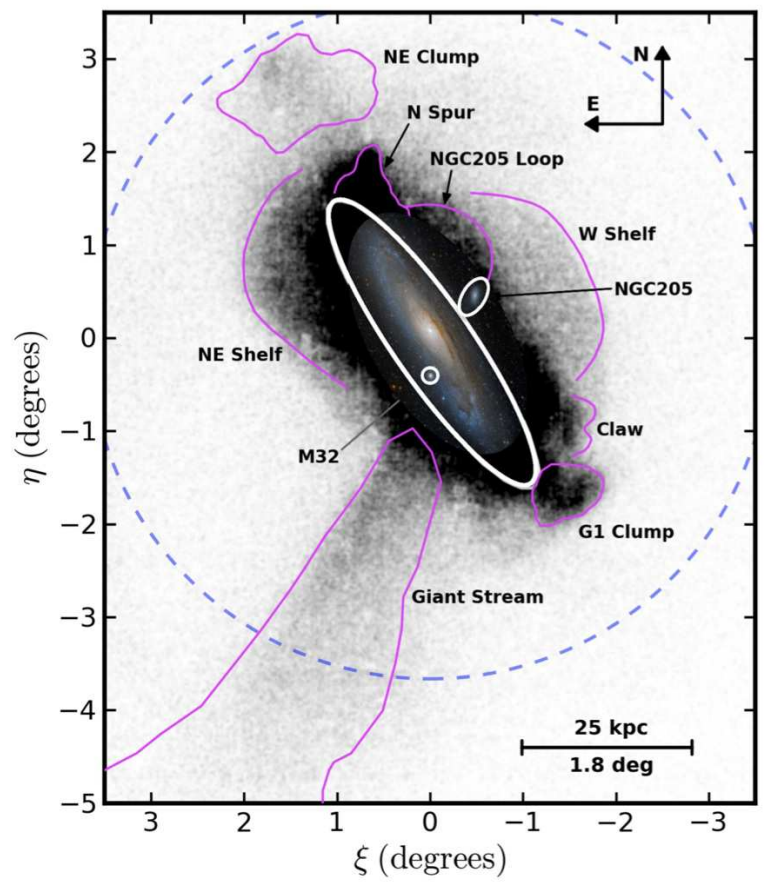

Fig. 2: The PAndAS map of metal-rich RGB stars in the inner halo of M31, upon which a typical textbook image of M31 is superposed. The map is constructed from stars with $i_{0} \leq 23.5$, having $-1 \leq[\mathrm{Fe} / \mathrm{H}] \leq 0$. The large white ellipse has a semimajor axis of $27 \mathrm{kpc}$ and delineates the full extent of the bright disk; the dashed blue circle has a radius of $50 \mathrm{kpc}$. Prominent inner halo substructure is outlined and labelled, as are the dwarf satellites M32 and NGC 205.

\subsection{The Giant Stellar Stream}

Over the last decade, the GSS has been the subject of intense study. On the observational side, efforts have concentrated on deriving quantities (e.g. distance, velocity) that can be used to model the orbit of the progenitor and on stellar populations constraints that can be used to establish its nature. On the theoretical side, work has focused on reconstructing the orbital history of the progenitor and using this knowledge to measure the halo mass of M31.

Line-of-sight distances to the stream are one of the key inputs for GSS orbit models. McConnachie et al. (2003) used measurements of the TRGB in a series of CFHT12K pointings to show that the GSS lies $\gtrsim 100 \mathrm{kpc}$ behind M31 at a projected radial distance of $60 \mathrm{kpc}$ and moves progressively closer to galaxy at smaller radii, with their distances being indistinguishable (within the uncertainties) at $\leq 10 \mathrm{kpc}$. 


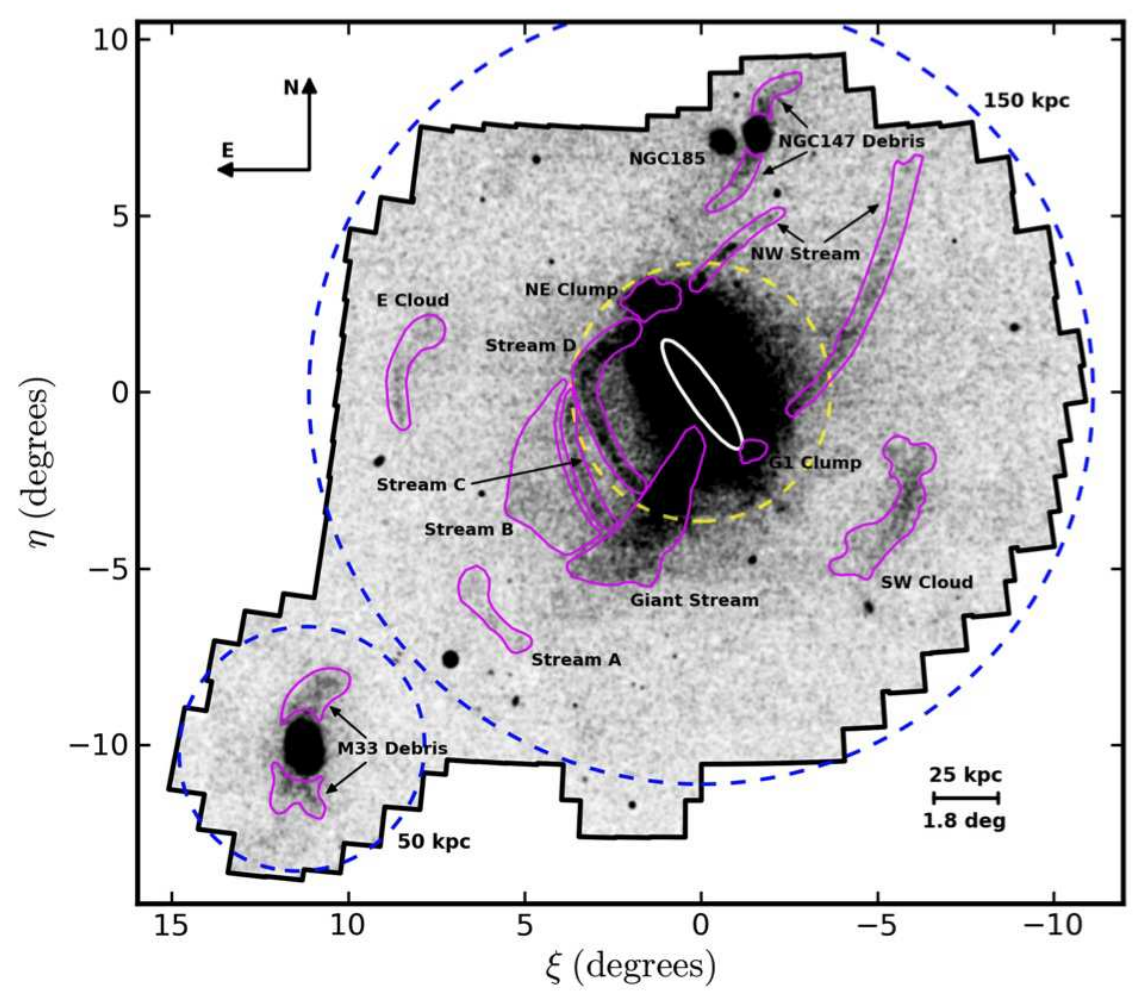

Fig. 3: A map of metal-poor RGB stars across the full extent of the PAndAS survey $\left(i_{0} \leq 23.5\right.$ and $\left.-2.5 \leq[\mathrm{Fe} / \mathrm{H}] \leq-1.1\right)$. The white ellipse is the same as in Fig. 2 while the dashed circles surrounding M 31 have radii of $50 \mathrm{kpc}$ (yellow) and $150 \mathrm{kpc}$ (blue) respectively and that surrounding M33 has a radius of $50 \mathrm{kpc}$. Prominent outer halo substructure is outlined and labelled, as are tidal streams associated with the satellite galaxies M33 and NGC 147.

These authors were also able to detect the stream in two fields on the northern side of M31, where it lies $\sim 40 \mathrm{kpc}$ in front of the galaxy, but no further, suggesting that the stream wraps fairly tightly around the M31 center towards the north-east.

Another key observable is the radial velocity distribution of stream stars. Individual RGB stars at the distance of M31 are sufficiently faint that an 8-m class telescope is required in order to measure their line-of-sight velocities; the DEIMOS multiobject spectrograph on the Keck II telescope has been the source of almost all the GSS radial velocity measurements to date (Ibata et al., 2004; Guhathakurta et al., 2006; Kalirai et al., 2006; Gilbert et al., 2009). These studies have shown that the radial velocity of the GSS becomes increasingly positive with increasing distance from the center of M31, ranging from $v_{\text {helio }}=-320 \mathrm{~km} \mathrm{~s}^{-1}$ at a projected dis- 

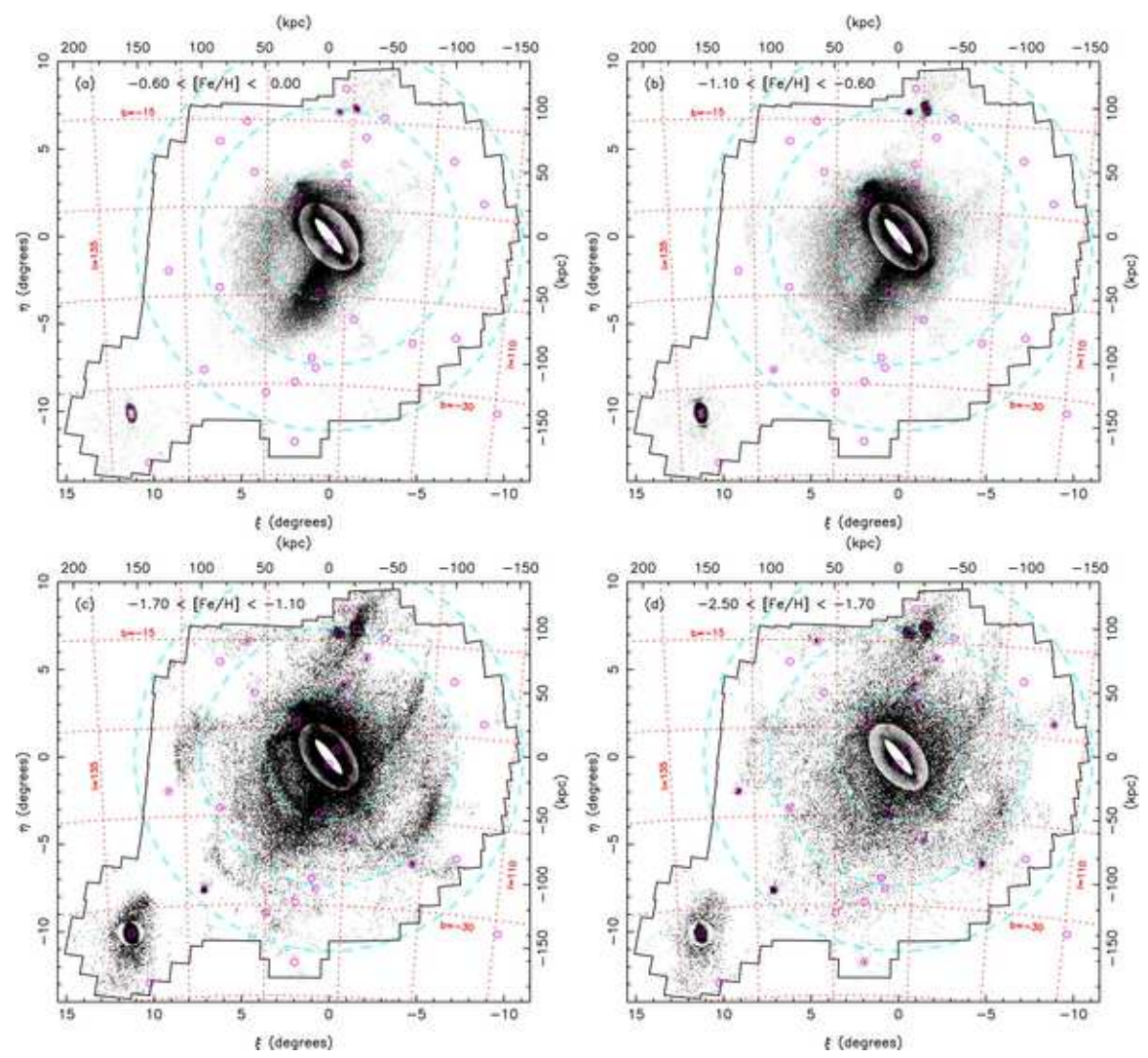

Fig. 4: The PAndAS map of RGB stars, as in Fig. 3, but presented this time as a function of metallicity. The two high metallicity bins (top panels) are dominated by the Giant Stellar Stream, although note that this structure changes morphology slightly between the two panels. While the low metallicity bottom left panel is dominated by numerous streams, the more metal-poor right-hand panel appears much smoother. Reproduced from Ibata et al. (2014).

tance of $60 \mathrm{kpc}$ to $v_{\text {helio }}=-524 \mathrm{~km} \mathrm{~s}^{-1}$ at a projected distance of $17 \mathrm{kpc}$. In all fields studied so far, the velocity dispersion is quite narrow - in the range of $10-30 \mathrm{~km} \mathrm{~s}^{-1}$. Intriguingly, a second cold kinematic component has been detected at several locations along the GSS (Kalirai et al., 2006; Gilbert et al., 2009). It has the same velocity gradient (and dispersion) as the primary GSS over the range in which the two have been mapped $(\sim 7 \mathrm{kpc})$ but it has a radial velocity that is offset by $\sim+100 \mathrm{~km} \mathrm{~s}^{-1}$. It is presently unclear whether this component is due to M31's disturbed disk or a forward wrap or bifurcation of the main stream.

The combination of line-of-sight distance and radial velocity measurements indicates that the GSS progenitor fell almost straight into M31 from behind. These 
data have motivated a variety of efforts to model the accretion of a dwarf satellite galaxy on a highly radial orbit (Ibata et al., 2004; Font et al., 2006; Fardal et al., 2006, 2008, 2013; Mori \& Rich, 2008; Sadoun et al., 2014). While these models differ in various aspects, they generally agree on the fact that the progenitor's initial stellar mass was in the range $1-5 \times 10^{9} \mathrm{M}_{\odot}$ and that its first pericentric passage came within a few kpc of the M31 center less than 1-2 Gyr ago. Some properties of the observed GSS, in particular the asymmetric distribution of stars along the stream cross section and the internal population gradient, are better reproduced in $N$-body models in which the progenitor possessed a rotating disk (e.g. Fardal et al., 2008; Sadoun et al., 2014).

In this near head-on collision, the progenitor experiences significant destruction at the first pericentric passage. Much of the satellite's mass is stripped off to form leading and trailing tidal streams, and a generic prediction is that much of M31's inner halo should be littered with this debris. Fig. 5 shows the sky distribution of debris in a set of recent $N$-body models of the GSS's orbit within the M31 potential (Fardal et al., 2013). Each panel differs in the adopted values of $M_{200}$, the M31 mass inside a sphere containing an average density 200 times the closure density of the universe, and $F_{p}$, the orbital phase of the progenitor at the present day. Although the exact pattern of debris depends on these (and a few other) parameters, all panels exhibit a similar morphology and show remarkable consistency with some of the features seen in Figs. 2 and 3. The GSS, the NE Shelf and the W Shelf are all naturally reproduced in this scenario, with the GSS representing the trailing stream of material torn off in the progenitor's first pericentric passage while the NE and $\mathrm{W}$ shelf regions contain material torn off in the second and third passages, respectively. These predictions are in excellent agreement with observations of the stellar populations of these substructures - which show striking similarities in their star formation histories (SFHs) and metallicity distributions to those of the GSS (e.g. Ferguson et al., 2005; Richardson et al., 2008) - as well as with the observed kinematics of the shelves (e.g. Gilbert et al., 2007; Fardal et al., 2012). The extensive pollution of M31's inner halo by material stripped from the GSS progenitor provides at least a partial explanation for why this region is dominated by more metalrich stellar populations compared to the Milky Way (e.g. Mould \& Kristian, 1986; Ibata et al., 2014).

Based on the projected positional alignment with the GSS, M31's luminous dwarf elliptical satellites M32 and NGC 205 were initially considered as prime candidates for the progenitor (Ibata et al., 2001; Ferguson et al., 2002). Indeed, both these systems possess unusual properties and, as will be discussed in Section 7 are tidally distorted in their outer regions (Choi et al., 2002; Ferguson et al., 2002). However, even the earliest attempts at orbit models ruled out a straightforward connection between the GSS progenitor and either of these two satellites (Ibata et al., 20042. Current models agree that any existing remnant should lie in the region of the NE Shelf, although thus far no candidate has been identified.

\footnotetext{
${ }^{2}$ Meanwhile, Block et al. 2006) argue that a head-on collision between M31 and M32 about 200 million years ago could be responsible for the formation of two off-center rings of ongoing star formation seen in the M31 disk.
} 
Although the location of the GSS progenitor remains a mystery, analysis of the stellar populations in the stream has provided important insight into its nature. Fig. 6]shows deep HST CMDs of a variety of tidal debris fields in the inner halo of M31, including those associated with the GSS. One can clearly see two predominant CMD morphologies - those which contain a narrow tilted red clump and a prominent horizontal branch extending quite far to the blue (labelled 'SL' in Fig. 6), and those which contain a rounder red clump, a well-populated blue plume and exhibit no horizontal branch (labelled 'DL' in Fig. 6). Those pointings which directly sample material associated with the GSS progenitor uniformly exhibit the morphology of the former type (Ferguson et al., 2005; Richardson et al., 2008). A third category, labelled ' $\mathrm{C}$ ', appears to be a composite of the previous morphologies.

Quantitative measures of the SFH and age-metallicity relation (AMR) of the GSS have been derived from deep CMDs using synthetic modelling techniques (Brown et al., 2006; Bernard et al., 2015a). The bottom panels of Fig. 7 show the most extensive analysis carried out to date, based on the 5 fields lying on GSS debris located throughout the inner halo. It appears that star formation in the progenitor got underway early on and at a fairly vigorous pace, peaking 8-9 Gyr ago. Star formation remained active until about 6 Gyr ago when there was a very rapid decline; this 'quenching' may indicate the time when the progenitor first entered the halo of M31. Roughly 50\% of the stellar mass in the GSS fields was in place $\sim 9$ Gyr ago, and the metallicity had reached the solar value by 5 Gyr ago, consistent with direct spectroscopy constraints from RGB stars (e.g. Guhathakurta et al., 2006; Kalirai et al., 2006; Gilbert et al., 2009). In addition, all the GSS fields probed thus far reveal a large spread in metallicity $(\geq 1.5 \mathrm{dex})$. Taken together, these properties suggest an early-type progenitor, such as a dwarf elliptical galaxy or spiral bulge. This further supports inferences from $N$-body modelling which suggest that the GSS progenitor was a fairly massive object with some degree of rotational support. Indeed, Fardal et al. (2013) argue it was likely the fourth or fifth most massive Local Group galaxy as recently as $1 \mathrm{Gyr}$ ago.

\subsection{Other Inner Halo Substructure}

While tidal debris from the GSS progenitor can explain the origin of some of the M31 inner halo substructure, other features require a different origin. In particular, the substructures lying near the major axis - such as the NE and G1 Clumps and the Claw - do not arise naturally in models of the dissolution of the stream progenitor.

A first step to understanding this low latitude substructure was taken by Ibata et al. (2005) who analysed the kinematics of stars in numerous fields around M31, including the G1 and NE Clumps. They noted a strong signature of rotation in almost all fields out to a galactocentric radius of $\sim 40 \mathrm{kpc}$, with some further detections out to $70 \mathrm{kpc}$. Stars are observed to move with velocities close to those expected for circular orbits in the plane of the M31 disk and with a typical velocity dispersion of 

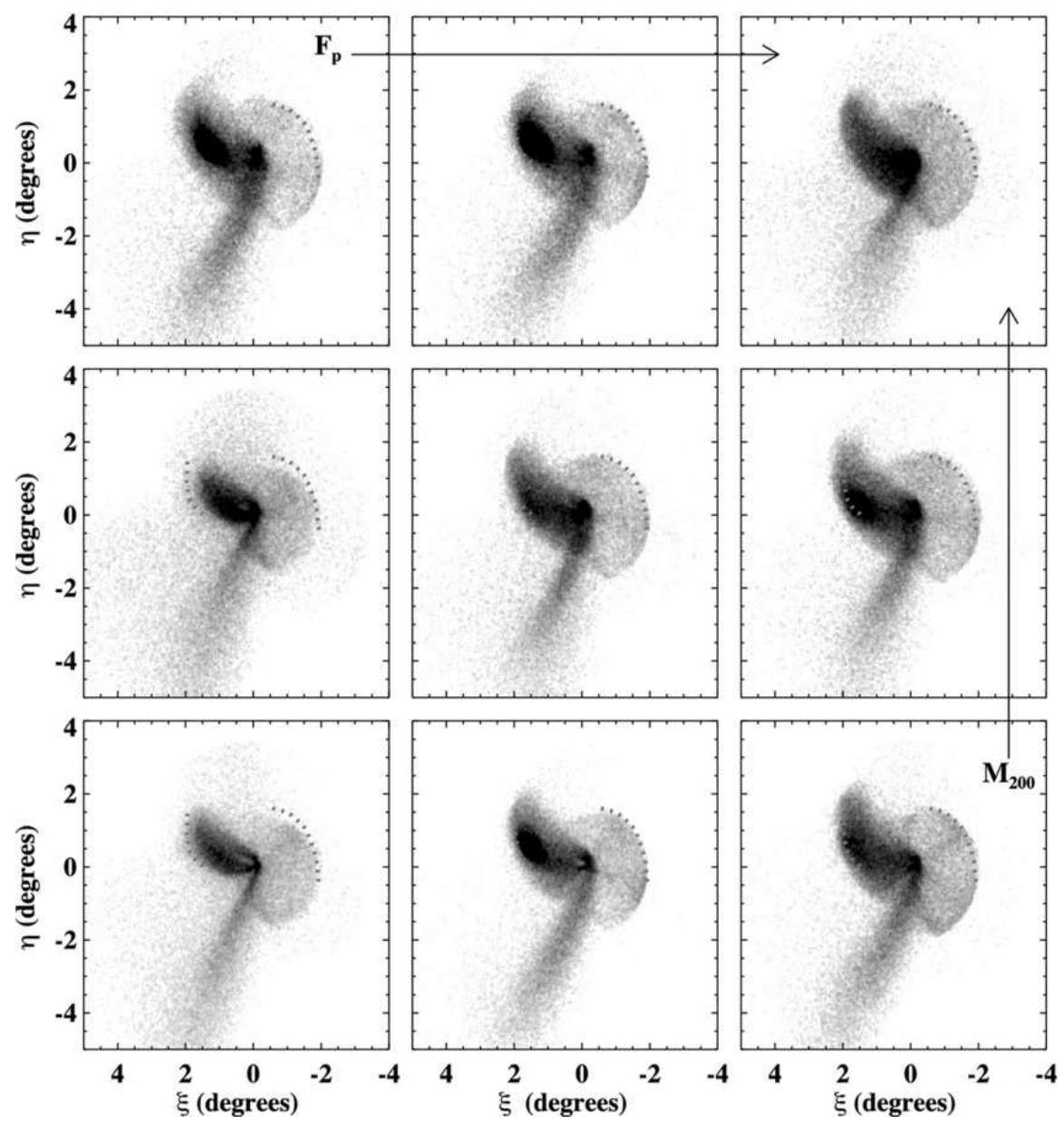

Fig. 5: Spatial distribution of stellar debris from the tidal disruption of the GSS progenitor, as predicted by a set of $9 \mathrm{~N}$-body simulations. The dashed lines show the observed boundaries of the $\mathrm{NE}$ and $\mathrm{W}$ shelves. In each case, different combinations of $M_{200}$, the virial mass of M31, and $F_{\mathrm{p}}$, the orbital phase of the progenitor at the present epoch, are adopted. While all panels share similar morphologies and resemble the main features seen in Figs.2] and3, some systematic differences can be seen as a function of the parameters. The present-day state of the progenitor varies from being tightly bound to highly dispersed; in all models shown here, it lies in the region of the NE shelf. Reproduced from Fardal et al. (2013).

$30 \mathrm{~km} \mathrm{~s}^{-1}$. Based on stacked spectra, Ibata et al. (2005) estimated the mean metallicity of these rotating outer populations to be $[\mathrm{Fe} / \mathrm{H}] \sim-0.9$. 
The irregular morphology yet coherent rotation observed in the outer disk regions led Ibata et al. (2005) to speculate that a vast disk-like structure was being assembled as a result of multiple accretion events. However, this interpretation faced a number of challenges, such as the homogeneity of the constituent stellar populations and the need for the accreted satellites to be sufficiently massive so that dynamical friction could circularise their orbits before disruption.

An alternative explanation is that much of the inner halo substructure is material that has either been torn off of the M31 outer disk or dynamically heated from the disk into the halo. It has long been known that the accretion of a low mass companion can have rather a disruptive effect on a stellar disk (e.g. Quinn et al., 1993; Walker et al., 1996) and more recent work has quantified the way in which disk stars can get ejected into the halo through such events (e.g. Zolotov et al., 2009; Purcell et al., 2010). Kazantzidis et al. (2008) demonstrated how the accretion of a population of satellites with properties drawn from a cosmological simulation can produce distinctive morphological features in the host galaxy's disk, similar to the inner halo substructures seen in M31. Additionally, they confirmed earlier work that showed the final distribution of disk stars exhibits a complex vertical structure that can be decomposed into a thin and thick disk (see also Villalobos \& Helmi, 2008).

Examination of the constituent stellar populations in the non-GSS debris fields supports the idea that this material has originated in the disk (e.g. Ferguson et al., 2005; Brown et al., 2006; Richardson et al., 2008; Bernard et al., 2015a). The 'DL' fields in Fig. 6 are rather homogeneous in appearance, all displaying a round red clump with significant luminosity width, a well-populated blue plume and no apparent horizontal branch - features that indicate continuous star formation and a moderately young mean age. The quantitative SFHs and AMRs of the 'disk-like' debris fields further strengthen this assertion; roughly $\sim 65 \%$ of the stars formed in the last $8 \mathrm{Gyr}$ and chemical evolution proceeded at a modest pace, starting from a pre-enriched level (see top panels of Fig. 7). Most importantly, these trends are strikingly similar to those that have been measured for populations in the M31 outer disk (Bernard et al., 2012, 2015b).

It is also notable that both the stream-like and disk-like fields in Fig. 7 show evidence for an enhancement in the rate of star formation roughly $2 \mathrm{Gyr}$ ago. This is surprising given that the constituent stellar populations in these fields have very different origins, and that many of them are substantially displaced from the main body (and the gas disk) of M31. There is now strong evidence that the M31 outer disk underwent a burst of star formation around this epoch, likely triggered by the relatively close passage of M33 (Bernard et al., 2012, 2015b). The existence of trace populations from this episode scattered throughout the inner halo, including up to $20 \mathrm{kpc}$ along the minor axis, argues for a redistribution of disk material in the intervening time. It would thus appear that, in addition to being heavily polluted by GSS debris, the M31 inner halo also contains a widespread component of heated disk stars (Bernard et al., 2015a). This idea was independently raised by Dorman et al. (2013) who find that a non-negligible fraction of the inner halo stars identified kinematically in M31 show a luminosity function consistent with an origin in the disk. 
Both the highly structured nature of the outer disk and the presence of displaced disk stars in the halo could be explained by one or more violent accretion events. Given the likely transitory nature of the outer disk substructures (Ibata et al., 2005) and the fact that stars as young as $2 \mathrm{Gyr}$ have been displaced into the halo, the event responsible for disrupting the disk must have been rather a recent one and it is tempting to speculate that it has been the head-on impact of the GSS progenitor roughly 1 Gyr ago (e.g. Mori \& Rich, 2008; Sadoun et al., 2014). If this scenario is correct, it implies that, in spite of its extremely messy appearance, all of M31's inner halo substructure can be traced to the direct and indirect effects of a single event.

\subsection{Outer Halo Substructure}

The outer halo debris features in M31 are much more poorly understood than those of the inner halo. While the inner halo is populated by tidal debris with a variety of morphologies and moderate metallicities, the outer halo is dominated by fairly narrow stellar streams and arcs which are, with the exception of the metal-rich component of Stream C, only apparent in maps constructed from metal-poor stars (see Fig. 37. The outer halo streams are of such low surface brightness that detailed characterisation of their stellar populations and kinematics has thus far been difficult. As will be discussed in the following section, GCs offer a very exciting way to probe the tidal features in these parts.

There are the four main structures visible in the far outer halo - Stream A, the E Cloud, the SW Cloud and the NW Stream. All of these features lie at radii $\gtrsim 100 \mathrm{kpc}$ from the center of M31 and subtend at least a few tens of kpc in length. Their CMDs indicate a similar metallicity of $[\mathrm{Fe} / \mathrm{H}] \sim-1.3$ (Ibata et al., 2007, 2014; Carlberg et al., 2011; Bate et al., 2014). The most luminous of the outer halo structures is the SW Cloud which Bate et al. (2014) estimate contains $\sim 5.6 \times 10^{6} \mathrm{~L} \odot$ or equivalently $M_{V} \approx-12.1$. This luminosity is approximately $75 \%$ of that expected for the feature on the basis of its measured metallicity and the Kirby et al. (2011) luminosity-metallicity relation. While this might indicate that a sizeable fraction of the luminosity of the parent object has been detected, it remains unclear at present whether these most distant halo features originate from distinct accretion events or material torn off from a single progenitor. Indeed, an interesting question is whether any of the outer halo debris can be traced to the accretion of the GSS progenitor. Although the metallicity of these features is considerably lower than that of the core of the GSS, it is a good match to that of the stream envelope (Ibata et al., 2007; Gilbert et al., 2009).

It is curious to note that the dwarf spheroidal satellite And XXVII appears projected on the upper segment of the NW Stream. Discovered by Richardson et al. (2011), this faint $\left(M_{V} \approx-7.9\right)$ system is highly morphologically disturbed and it is tempting to speculate that it may be the source of the NW Stream debris. However, the metallicity of the stream stars appears somewhat higher than that of the dwarf 
galaxy which complicates the interpretation Carlberg et al., 2011; Collins et al., 2013). Furthermore, Collins et al. (2013) note additional kinematic substructure in the vicinity of And XXVII which is not yet understood.

Ibata et al. (2014) have recently conducted a global analysis of the large-scale structure of the M31 halo using data from the PAndAS survey. Despite the presence of copious substructures throughout, they find that the stellar halo populations closely follow power-law profiles that become steeper with increasing metallicity. The smooth metal-poor halo component (defined as the population with $[\mathrm{Fe} / \mathrm{H}]<-1.7$ that cannot be resolved into spatially distinct substructures with PAndAS), has a global (3D) power-law slope of $\gamma=-3.08 \pm 0.07$ and an almost spherical shape, but accounts for a mere $\sim 5 \%$ of the overall halo luminosity. By far, most of the luminosity of the halo out to the edge of the PAndAS survey resides in moderate-metallicity substructure. Ibata et al. (2014) estimate that the total stellar mass of the M31 halo at distances beyond $2^{\circ}$ is $\sim 1.1 \times 10^{10} \mathrm{M}_{\odot}$ and that the mean metallicity decreases from $[\mathrm{Fe} / \mathrm{H}]=-0.7$ at $\mathrm{R}=30 \mathrm{kpc}$ to $[\mathrm{Fe} / \mathrm{H}]=-1.5$ at $\mathrm{R}=150 \mathrm{kpc}$ for the full sample. An alternative approach to studying the outer halo in M31 has been taken by the SPLASH team who have obtained spectroscopy of RGB stars in many pencil-beam fields extending out to $175 \mathrm{kpc}$ (Guhathakurta et al., 2006; Kalirai et al., 2006; Gilbert et al., 2006, 2012, 2014). In contrast to photometric studies, their approach allows them to identify and remove kinematic substructure in their fields, at least out to $90 \mathrm{kpc}$. Although it is not possible to directly compare the results of the PAndAS and SPLASH surveys, which are based on very different sample selections, their inferences on the global halo properties of M31 appear to be largely consistent (Ibata et al., 2014; Gilbert et al., 2014).

\section{Globular Clusters as Probes of Tidal Streams in M31}

An intriguing question regarding the substructures seen in the halo of M31 is whether they show any degree of spatial correlation with members of the M31 GC system. This is motivated in part by the long-held suspicion that a substantial number of the GCs in the Milky Way halo did not form in situ, but rather in small satellite dwarf galaxies that subsequently fell into the Galactic potential well and disintegrated. This idea was first suggested in the seminal paper by Searle \& Zinn (1978) and was spectacularly verified in the early 1990s with the discovery of the disrupting Sagittarius dwarf, which is in the process of depositing at least five GCs into the outer halo of the Milky Way (e.g. Bellazzini. Ferraro, \& Ibata, 2003; Law \& Majewski, 2010). Modern studies of the Galactic GC system have only served to add further weight to the assertion - it is now known that the abundances, velocities, ages, horizontal branch morphologies, and sizes of perhaps up to a third of Milky Way GCs are consistent with an external origin (e.g., Zinn, 1993; Mackey \& Gilmore, 2004; Mackev \& van den Bergh, 2005; Marín-Franch et al., 2009; Forbes \& Bridges, 2010; Dotter et al., 2010, 2011). 
Historically almost all work on the M31 GC system has been confined to regions comparatively close to the galactic center, typically within $\mathrm{R} \sim 20-25 \mathrm{kpc}$; however the situation has changed thanks to the aforementioned wide-field mapping surveys of M31. In particular, the INT/WFC and PAndAS surveys, but also to some extent the SDSS, have facilitated the first detailed and uniform census of the outer halo GC system of M31 (Huxor et al., 2005, 2008, 2011, 2014; Martin et al., 2006; di Tullio Zinn \& Zinn, 2013, 2014). Remote clusters are abundant in M31; there are now more than 90 objects known to reside at projected radii beyond 25 $\mathrm{kpc}, 13$ of which sit outside $100 \mathrm{kpc}$ in projection, with the most the most distant at $\sim 140 \mathrm{kpc}$ in projection and up to $200 \mathrm{kpc}$ in three dimensions (Mackey et al., 2010a, 2013b). This is many more than is seen in the outskirts of the Milky Way while the disparity in the number of GCs in the Milky Way and M31 within $\sim 25$ kpc is roughly 3:1 in favour of M31, outside this radius it is more like 7:1 in favour of M31 (Huxor et al., 2014).

Figure 8 shows the positions of all known M31 GCs plotted on top of the PAndAS spatial density map of metal-poor RGB stars. In the outer parts of the halo, where large, coherent tidal debris streams are readily distinguished, there is a striking correlation between these features and the positions of a large fraction $(\sim 50-80 \%)$ of the GCs. Substantial numbers of clusters are seen projected onto the NW Stream, the SW Cloud, the E Cloud, and the overlapping portion of Streams C and D. There is, in addition, a statistically significant overdensity of clusters ("Association 2") sitting near the base of the NW Stream, that cannot (as of yet) be identified with a visible tidal stream in the field halo.

Mackey et al. (2010b) have demonstrated that the probability of this global alignment between clusters and streams arising randomly is low - well below $1 \%$ for a GC system possessing an azimuthally uniform spatial distribution. This implies that the observed coincidence represents a genuine physical association and hence direct evidence that much of the outer M31 GC system has been assembled via accretion. Moreover, at least some of the properties of the accreted M31 GCs appear to be consistent with those exhibited by ostensibly accreted Galactic members - particular examples being those of younger ages (Mackey et al., 2013a) and extended structures (Huxor et al., 2011; Tanvir et al., 2012).

The argument made by Mackey et al. (2010b) is based entirely on statistical grounds; to determine on an object-by-object basis which GCs are associated (or not) with a given substructure requires kinematic information. The most extensive kinematic study of the M31 outer halo GC system to date is by Veljanoski et al. (2013, 2014), who acquired spectra for 71 clusters outside a projected radius of 30 kpc (representing $86 \%$ of the known population in these parts); the velocities of these objects in the M31-centric frame are color-coded in Fig. 8 It can be readily seen that GCs projected onto a given outer halo substructure tend to exhibit correlated velocities (see left panel of Fig. 9 for an example). Those objects on the NW Stream and SW Cloud reveal strong velocity gradients from one end of the substructure to the other, while clusters on the E Cloud form a close group in phase space. Members of the Stream C/D overlap area, and those in Association 2 split into additional sub-groups by velocity. A remarkable feature of many of the ensem- 
bles considered by Veljanoski et al. (2014) is the coldness of their kinematics, with all GC groupings exhibiting velocity dispersions consistent with zero given the individual measurement uncertainties. These results strongly reinforce the notion that a substantial fraction of the outer halo GC population of M31 has been accreted, and that these clusters trace the velocities of the tidal streams from their progenitor systems. Indeed, while definitive measurements have thus far only been possible for two substructures, the velocities of the GCs sitting on Stream C and on the SW Cloud have been shown to be in excellent agreement with those of the underlying stream stars (Collins et al., 2009; Mackey et al., 2014).

Fig. 8 also demonstrates the surprising result that the M31 GC system as a whole possesses bulk rotation - those GCs to the west of M31 appear to systematically possess negative velocities in the M31-centric frame, while those to the east typically have positive velocities. Veljanoski et al. (2014) compared a variety of kinematic models to the data and found a rotation amplitude of $86 \pm 17 \mathrm{~km} \mathrm{~s}^{-1}$ around an axis aligned with the M31 optical minor axis provided the best match. This rotation velocity is quite substantial - for comparison, Velianoski et al. (2014) also found evidence that the velocity dispersion in the cluster system decreases from $129_{-24}^{+22} \mathrm{~km} \mathrm{~s}^{-1}$ at $30 \mathrm{kpc}$ to roughly $75 \mathrm{~km} \mathrm{~s}^{-1}$ at $100 \mathrm{kpc}$. The right panel of Figure 9 further elucidates this rotation by showing the GC velocities in the M31-centric frame as a function of projected radius along the major axis. It is apparent that the rotation of the outer halo GC system is in the same sense as for the inner halo clusters (and indeed the M31 disk), albeit with smaller amplitude. Importantly, the rotation does not seem to be driven purely by clusters that sit on the particular halo substructures nor by those sitting well away from any underlying halo feature - both groups apparently share in the pattern equally.

Understanding the origin of the angular momentum in the outer halo GC system of M31 presents a significant challenge. One possibility is if a large fraction of the halo GC system was brought into M31 by just one relatively massive host galaxy on a low inclination orbit. However, in this scenario it is difficult to explain the observed presence of distinct dynamically cold subgroups of GCs as well as the typically narrow stellar debris streams in the halo. Another possibility is that the outer halo GC system results from the assimilation of several dwarf galaxies, but that these were accreted onto M31 from a preferred direction on the sky. This scenario might well be related to the recent discovery that many dwarf galaxies, both in the Milky Way and M31, appear to lie in thin rotating planar configurations such that their angular momenta are correlated (Ibata et al., 2013; Pawlowski. Pflamm-Altenburg. \& Kroupa, 2012). In this context it is relevant that almost all of the dwarf galaxies thought to be members of the planes presently observed in both M31 and the Milky Way are insufficiently massive to host GCs, and furthermore that the rotation axes of the M31 outer halo GC system and the M31 dwarf plane are misaligned with each other by $\sim 45^{\circ}$. 


\section{Tidal Streams from M31's Satellite Galaxies}

M31 has a substantially richer satellite system than the Milky Way. Not only does it possess more dwarf companions, but it also possesses several moderately luminous ones: M33 with $M_{\mathrm{V}}=-18.8$ and the four dwarf ellipticals (dEs) M32, NGC 205, NGC 147 and NGC 185 with $M_{\mathrm{V}}=-15.5$ to -16.5 (McConnachie, 2012; Crnojević et al., 2014). Tidal features are now known around nearly all of these systems, indicating that they are in the process of depositing tidally-stripped stars into the M31 halo.

The innermost satellites of M31 are M32 and NGC 205, which lie at M31-centric distances of $25 \mathrm{kpc}$ and $42 \mathrm{kpc}$, respectively (McConnachie, 2012). These systems overlap the main body of M31 in projection, a fact that has greatly complicated detailed analyses of their outer structures. Nonetheless, diffuse light studies indicate that both systems exhibit breaks in their surface brightness profiles which are accompanied by sharp changes in isophotal ellipticity and position angle; such behaviour is consistent with expectations for systems undergoing tidal interaction and stripping (Choi et al., 2002; Johnston et al., 2002). Additionally, Ferguson et al. (2002) present isopleth maps from a scanned deep photographic plate which reveal the characteristic " $\mathrm{S}$ "-shape of tidal distortion in their peripheral regions. It is therefore curious that kinematic studies support the existence of unbound stars at large radii in NGC 205 but not in M32 (Geha et al., 2006; Howley et al., 2013).

Using resolved star count data from the INT/WFC survey, McConnachie et al. (2004) detected a $15 \mathrm{kpc}$ long stellar arc that emanates from the northern side of NGC 205 before bending eastward back to the M31 disk. This discovery resulted from the fact that the stellar loop consists of RGB stars that are slightly bluer than the M31 inner halo stars and hence have enhanced contrast on metal-poor star count maps. Unfortunately, the true nature of this feature remains controversial. While McConnachie et al. (2004) detected a kinematic feature centered at $-160 \mathrm{~km} \mathrm{~s}^{-1}$ with a dispersion of $10 \mathrm{~km} \mathrm{~s}^{-1}$ that they attributed to the NGC 205 loop, subsequent re-examination of the data by Ibata et al. (2005) did not recover this cold component. Instead, they argued that the kinematic properties of this feature were consistent with the bulk motion of M31's disk. Furthermore, Richardson et al. (2008) and Bernard et al. (2015a) find that deep HST CMDs of this feature can be explained naturally by a combination of heated disk and GSS debris, without requiring any additional component of stars, while Howley et al. (2008) find the most likely orbit of NGC 205 to be incompatible with the location of the arc.

More recently, data from the the PAndAS survey has been used to explore the outer regions of the dE satellites NGC 147 and NGC 185 to extremely faint surface brightness levels using resolved stars. Projected $\sim 100 \mathrm{kpc}$ north of M31, and lying at M31-centric distances of $120 \mathrm{kpc}$ and $180 \mathrm{kpc}$, respectively (McConnachie, 2012), these systems are sufficiently remote that contamination from M31 itself is minimal and the main complication is the presence of substantial foreground Galactic populations along their sightlines. Crnojević et al. (2014) trace both systems to

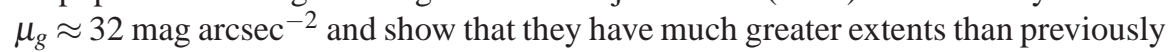
recognized. As can be seen in Figs. 3 and 4, NGC 185 retains a regular shape in its 
peripheral regions while NGC 147 exhibits pronounced isophotal twisting due to the emergence of long symmetric tidal tails. Even neglecting these tails, NGC 147 appears more distended with an effective radius almost three times that of NGC 185. In contrast to NGC 185, it also exhibits no metallicity gradient. These differences in the structure and stellar populations of the dEs suggest that tidal influences have played an important role in governing the evolution of NGC 147, but not NGC 185. On the assumption that NGC 147, NGC 185 and nearby dwarf spheroidal CassII form a bound subgroup, Arias et al. (2016) show that it is possible to find orbits around M31 which result in substantial tidal disruption to NGC 147 but not the other two systems.

M31's most massive satellite is the low-mass spiral M33, lying almost $210 \mathrm{kpc}$ away (McConnachie, 2012). Although no unusual structure was detected around M33 in the early INT/WFC survey (Ferguson et al., 2007), the deeper PAndAS data led to the discovery of a gigantic "S"-shaped substructure that surrounds the main body of the galaxy (McConnachie et al., 2009, 2010). Traced to a projected radius of $40 \mathrm{kpc}$ and $\mu_{g} \approx 33 \mathrm{mag} \operatorname{arcsec}^{-2}$, this feature coincides with a similar feature that was detected in H I (Putman et al., 2009). McConnachie et al. (2009) used $N$-body simulations to conduct a preliminary exploration of the idea that this is the signature of M33's tidal disruption as it orbits around M31. They found that a relatively close recent encounter could explain the appearance of this low surface brightness substructure while satisfying the known phase space constraints of the two systems. Specifically, they suggest that M31 and M33 came within 40-50 kpc of each other roughly $2.5 \mathrm{Gyr}$ ago, a hypothesis which is also consistent with the more recent Local Group orbit modelling work of van der Marel et al. (2012). This timescale is particularly interesting since the outer disks of both systems appear to have experienced strong bursts of star formation at this time and there is evidence to suggest the inflow of metal-poor gas (Bernard et al., 2012, 2015b). Thus, while more detailed modelling of this interaction is clearly required, there is tantalising evidence to suggest that it could explain a number of puzzling aspects about the M31-M33 system, such as the strong warps in both galaxies and the unusual synchronous burst of star formation.

\section{Summary and Future Prospects}

The vast amount of work on the M31 halo over the past 15 years has led to an exceptional situation where, in many ways, our knowledge of the peripheral regions of that system far exceeds our knowledge of those regions in our own Milky Way. Studies have been able to identify and characterise coherent debris streams in the M31 halo out to projected galactocentric distances of $\sim 120 \mathrm{kpc}$, and detect and map the properties of the 'smooth' halo out to $\sim 150-200 \mathrm{kpc}$. Even in the inner halo ( $\$ 50 \mathrm{kpc}$ ), we have a much clearer understanding of the nature and origin of the tidal debris, thanks to our external perspective. This can be contrasted with the situation in the Milky Way where many of the inner halo/outer disk structures are 
very poorly understood (e.g. the Monoceros, Hercules-Aquila, Tri-And, and Virgo overdensities; see Chapters $3 \& 4$ ).

In comparison to the Milky Way, the M31 inner halo is littered with metal-rich debris. Some of this material has been stripped from the GSS progenitor, while the rest is likely to be material from the M31 disk heated by the recent impact of this satellite. M31 may also have more outer halo tidal streams than the Milky Way, although most Milky Way surveys to date have not had the sensitivity to detect substructures in these parts. Additionally, M31 (1) has a higher fraction of its total light in the halo component compared to the Milky Way (e.g. Ibata et al., 2014; Gilbert et al., 2012), (2) is characterised by a smooth density profile unlike the broken power-law of the Milky Way (Deason et al., 2013) and (3) has a substantially larger population of halo GCs, many of which lie along streams (Mackey et al., 2010b; Veljanoski et al., 2014). It is likely that these differences result from the unique accretion histories experienced by the two systems. M31 may have experienced more accretions than the Milky Way, or it may simply have experienced a more prolonged history of accretion. Indeed, there is a tantalising similarity between the properties of the Sagittarius dwarf and those inferred for the GSS progenitor - both are early-type galaxies with estimated initial masses in the range $0.5-1 \times 10^{9} \mathrm{M}_{\odot}$. The major building blocks of these halos may well have been comparable, but their orbits and accretion times rather different. In this spirit, it is interesting to speculate how the Milky Way and M31 halos would have compared to each other $\sim 2$ Gyr ago, before the GSS progenitor entered M31's inner halo.

A number of outstanding questions remain regarding the M31 system, and much exciting progress can be expected over the next decade. Some of the most pressing issues that remain to be addressed include:

- The development of a definitive understanding of M31's most significant recent accretion event: what was the true nature of the GSS progenitor and where is the remnant now? How did it come to be on such an extreme orbit and how did it survive in this state until $\sim 1$ Gyr ago? What is the explanation for the second velocity component in the GSS and is there any connection between the GSS progenitor and the similarly metal-rich component of Stream C?

- Understanding the origin of the outer halo debris. What and where are the progenitors of the outer halo debris streams? Do these features result from one or many accretion events? The associated GCs are likely to play an important role in answering these questions, and also in using these streams to derive refined estimates of the mass and potential of M31. While line-of-sight distances to stream GCs are possible with HST observations, proper motion measurements may be possible for the most compact objects with Gaia.

- Understanding the origin of the coherent rotation in the outer GC population, and how it can be reconciled with the supposedly chaotic accretion of parent dwarf galaxies into the halo. Additionally, do the underlying debris streams and the smooth field halo also exhibit this rotation? Given the sparse nature of the stellar populations in these parts, very large-scale spectroscopy campaigns covering a significant fraction of the halo will be required to answer these questions. 
- Developing a holistic picture of M31's evolution that links its accretion history to its global evolution and current structure. There are fascinating hints that the recent interaction and accretion history of M31 can explain a variety of puzzling observations. The close passage of M33 could excite the strong asymmetric warps and bursts of star formation observed in both systems, while the accretion of the GSS progenitor could further disrupt the M31 outer disk, displace some fraction of the disk stars into the halo and deposit a substantial amount of metal rich debris in the inner halo. Much work is required to verify and fine-tune these ideas and there is a particular need for further detailed $N$-body modelling.

- Searching for the edge of the M31 stellar halo. Tidal streams, field stars and GCs have been found to the very edge of the PAndAS survey, suggesting that they could extend yet further. New wide-field mapping facilities such as HyperSuprime Cam on the Subaru telescope will be required to efficiently explore beyond the limit of PAndAS and may benefit from the use of specialised filters to discriminate between foreground and M31 populations.

Acknowledgements We thank Edouard Bernard and Jovan Veljanoski for their help in creating Figs. 7 and 8 AMNF acknowledges support from an STFC Consolidated Grant (ST/J001422/1) and the hospitality of the Instituto de Astrofisica de Canarias while completing this chapter. ADM is grateful for support from the Australian Research Council through Discovery Projects DP1093431, DP120101237 and DP150103294.

\section{References}

Arias, V., Guglielmo, M., Nuwanthika, N., et al. 2016, MNRAS, 456, 1654

Baade, W., \& Gaposchkin, C. H. P. 1963, Evolution of stars and galaxies., Cambridge, Harvard University Press.

Bate, N. F., Conn, A. R., McMonigal, B., et al. 2014, MNRAS, 437, 3362

Bellazzini, M., Ferraro, F. R., Ibata, R. A. 2003, A. J., 125, 188

Belokurov, V., Zucker, D. B., Evans, N. W., et al. 2006, ApJ, 642, L137

Bernard, E. J., Ferguson, A. M. N., Barker, M. K., et al. 2012, MNRAS, 420, 2625

Bernard, E. J., Ferguson, A. M. N., Richardson, J. C., et al. 2015a, MNRAS, 446, 2789

Bernard, E. J., Ferguson, A. M. N., Chapman, S. C. et al. 2015b, MNRAS, 453, L113

Block, D. L., Bournaud, F., Combes, F., et al. 2006, Nature, 443, 832

Brown, T. M., Smith, E., Ferguson, H. C., et al. 2006, Ap. J., 652, 323

Carlberg, R. G., Richer, H. B., McConnachie, A. W., et al. 2011, Ap. J., 731, 124

Chapman, S. C., Ibata, R., Irwin, M., et al. 2008, MNRAS, 390, 1437

Choi, P. I., Guhathakurta, P., \& Johnston, K. V. 2002, A. J., 124, 310

Ciardullo, R., Jacoby, G. H., Ford, H. C., \& Neill, J. D. 1989, Ap. J., 339, 53

Coccato, L., Arnaboldi, M., \& Gerhard, O. 2013, MNRAS, 436, 1322

Collins, M. L. M., Chapman, S. C., Rich, R. M., et al. 2013, Ap. J., 768, 172

Collins, M. L. M., Chapman, S. C., Irwin, M. J., et al. 2009, MNRAS, 396, 1619

Cram, T. R., Roberts, M. S., \& Whitehurst, R. N. 1980, A\&AS, 40, 215

Crnojević, D., Ferguson, A. M. N., Irwin, M. J., et al. 2014, MNRAS, 445, 3862

Crotts, A. P. S. 1986, A. J., 92, 292

Deason, A. J., Belokurov, V., Evans, N. W., \& Johnston, K. V. 2013, Ap. J., 763, 113

Diaz, J. D., Koposov, S. E., Irwin, M., Belokurov, V., \& Evans, N. W. 2014, MNRAS, 443, 1688 
di Tullio Zinn, G., \& Zinn, R. 2013, A. J., 145, 50

di Tullio Zinn, G., \& Zinn, R. 2014, A. J., 147, 90

Dotter, A., Sarajedini, A., Anderson, J., et al. 2010, Ap. J., 708, 698

Dotter, A., Sarajedini, A., Anderson, J. 2011, Ap. J., 738, 74

Dorman, C. E., Widrow, L. M., Guhathakurta, P., et al. 2013, Ap. J., 779, 103

Fardal, M. A., Babul, A., Geehan, J. J., \& Guhathakurta, P. 2006, MNRAS, 366, 1012

Fardal, M. A., Guhathakurta, P., Babul, A., \& McConnachie, A. W. 2007, MNRAS, 380, 15

Fardal, M. A., Babul, A., Guhathakurta, P., Gilbert, K. M., \& Dodge, C. 2008, Ap. J. Lett., 682, L33

Fardal, M. A., Guhathakurta, P., Gilbert, K. M., et al. 2012, MNRAS, 423, 3134

Fardal, M. A., Weinberg, M. D., Babul, A., et al. 2013, MNRAS, 434, 2779

Faria, D., Johnson, R. A., Ferguson, A. M. N., et al. 2007, AJ, 133, 1275

Ferguson, A. M. N., Irwin, M. J., Ibata, R. A., Lewis, G. F., \& Tanvir, N. R. 2002, A. J., 124, 1452

Ferguson, A. M. N., Johnson, R. A., Faria, D. C., et al. 2005, Ap. L. Lett., 622, L109

Ferguson, A., Irwin, M., Chapman, S., et al. 2007, Island Universes - Structure and Evolution of Disk Galaxies, 239

Font, A. S., Johnston, K. V., Guhathakurta, P., Majewski, S. R., \& Rich, R. M. 2006, A. J., 131, 1436

Forbes, D. A., Bridges, T. 2010, MNRAS, 404, 1203

Foster, C., Lux, H., Romanowsky, A. J., et al. 2014, MNRAS, 442, 3544

Gebhardt, K., Rich, R. M., \& Ho, L. C. 2005, Ap. J., 634, 1093

Geha, M., Guhathakurta, P., Rich, R. M., \& Cooper, M. C. 2006, A. J., 131, 332

Gilbert, K. M., Guhathakurta, P., Kalirai, J. S., et al. 2006, Ap. J., 652, 1188

Gilbert, K. M., Fardal, M., Kalirai, J. S., et al. 2007, Ap. J., 668, 245

Gilbert, K. M., Guhathakurta, P., Kollipara, P., et al. 2009, Ap. J., 705, 1275

Gilbert, K. M., Guhathakurta, P., Beaton, R. L., et al. 2012, Ap. J., 760, 76

Gilbert, K. M., Kalirai, J. S., Guhathakurta, P., et al. 2014, Ap. J., 796, 76

Guhathakurta, P., Rich, R. M., Reitzel, D. B., et al. 2006, A. J., 131, 2497

Howley, K. M., Geha, M., Guhathakurta, P., et al. 2008, Ap. J., 683, 722

Howley, K. M., Guhathakurta, P., van der Marel, R., et al. 2013, Ap. J., 765, 65

Huxor, A. P., Tanvir, N. R., Irwin, M. J., et al. 2005, MNRAS, 360, 1007

Huxor, A. P., Tanvir, N. R., Ferguson, A. M. N., et al. 2008, MNRAS, 385, 1989

Huxor, A. P., Ferguson, A. M. N., Tanvir, N. R., et al. 2011, MNRAS, 414, 770

Huxor, A. P., Mackey, A. D., Ferguson, A. M. N., et al. 2014, MNRAS, 442, 2165

Ibata, R., Irwin, M., Lewis, G., Ferguson, A. M. N., \& Tanvir, N. 2001, Nature, 412, 49

Ibata, R., Chapman, S., Ferguson, A. M. N., et al. 2004, MNRAS, 351, 117

Ibata, R., Chapman, S., Ferguson, A. M. N., et al. 2005, Ap. J., 634, 287

Ibata, R., Martin, N. F., Irwin, M., et al. 2007, Ap. J., 671, 1591

Ibata, R. A., Lewis, G. F., Conn, A. R., et al. 2013, Nature, 493, 62

Ibata, R. A., Lewis, G. F., McConnachie, A. W., et al. 2014, Ap. J., 780, 128

Innanen, K. A., Kamper, K. W., van den Bergh, S., \& Papp, K. A. 1982, Ap. J. , 254, 515

Irwin, M. J., Ferguson, A. M. N., Ibata, R. A., Lewis, G. F., \& Tanvir, N. R. 2005, Ap. J. Lett., 628, L105

Irwin, M. J., Ferguson, A. M. N., Huxor, A. P., et al. 2008,Ap. J., 676, L17

Johnston, K. V., Choi, P. I., \& Guhathakurta, P. 2002, A. J., 124, 127

Kalirai, J. S., Guhathakurta, P., Gilbert, K. M., et al. 2006, Ap. J., 641, 268

Kazantzidis, S., Bullock, J. S., Zentner, A. R., Kravtsov, A. V., \& Moustakas, L. A. 2008, Ap. J., 688, 254

Kirby, E. N., Lanfranchi, G. A., Simon, J. D., Cohen, J. G., \& Guhathakurta, P. 2011, Ap. J., 727, 78

Kniazev, A. Y., Grebel, E. K., Zucker, D. B., et al. 2014, A. J., 147, 16

Law, D. R., Majewski, D. R. 2010, Ap. J., 718, 1128

Lewis, G. F., Braun, R., McConnachie, A. W., et al. 2013, Ap. J., 763, 4

Mackey, A. D., Gilmore, G. F. 2004, MNRAS, 355, 504 
Mackey, A. D., van den Bergh, S. 2005, MNRAS, 360, 631

Mackey, A. D., Ferguson, A. M. N., Irwin, M. J., et al. 2010a, MNRAS, 401, 533

Mackey, A. D., Huxor, A. P., Ferguson, A. M. N., et al. 2010b, Ap. J. Lett., 717, L11

Mackey, A. D., Huxor, A. P., Ferguson, A. M. N., et al. 2013a, MNRAS, 429, 281

Mackey, A. D., Huxor, A. P., Martin, N. F., et al. 2013b, Ap. J. Lett., 770, L17

Mackey, A. D., Lewis, G. F., Collins, M. L. M., et al. 2014, MNRAS, 445, L89

Marín-Franch, A., Aparicio, A., Piotto, G., et al. 2009, Ap. J., 694, 1498

Martin, N. F., Ibata, R. A., Irwin, M. J., et al. 2006, MNRAS, 371, 1983

McConnachie, A. W., Irwin, M. J., Ibata, R. A., et al. 2003, MNRAS, 343, 1335

McConnachie, A. W., Irwin, M. J., Lewis, G. F., et al. 2004,MNRAS, 351, L94

McConnachie, A. W., Irwin, M. J., Ferguson, A. M. N., et al. 2005, MNRAS, 356,979

McConnachie, A. W., Irwin, M. J., Ibata, R. A., et al. 2009, Nature, 461, 66

McConnachie, A. W., Ferguson, A. M. N., Irwin, M. J., et al. 2010, Ap. J., 723, 1038

McConnachie, A. W. 2012, A.J., 144, 4

Merrett, H. R., Kuijken, K., Merrifield, M. R., et al. 2003, MNRAS, 346, L62

Merrett, H. R., Merrifield, M. R., Douglas, N. G., et al. 2006, MNRAS, 369, 120

Meylan, G., Sarajedini, A., Jablonka, P., et al. 2001,A. J., 122, 830

Morris, P. W., Reid, I. N., Griffiths, W. K., \& Penny, A. J. 1994, MNRAS, 271, 852

Mori, M., \& Rich, R. M. 2008, Ap. J. Lett., 674, L77

Morrison, H. L., Harding, P., Hurley-Keller, D., \& Jacoby, G. 2003, Ap. J. Lett., 596, L183

Mould, J., \& Kristian, J. 1986, Ap. J., 305, 591

Newberg, H. J., Yanny, B., Grebel, E. K., et al. 2003, Ap. J. Lett., 596, L191

Newton, K., \& Emerson, D. T. 1977, MNRAS, 181, 573

Ostheimer, J. C., Jr. 2003, Ph.D. Thesis,

Pawlowski, M. S., Pflamm-Altenburg, J., Kroupa, P. 2012, MNRAS, 423, 1109

Pritchet, C. J., \& van den Bergh, S. 1988, Ap. J., 331, 135

Pritchet, C. J., \& van den Bergh, S. 1994, A.J., 107, 1730

Purcell, C. W., Bullock, J. S., \& Kazantzidis, S. 2010, MNRAS, 404, 1711

Putman, M. E., Peek, J. E. G., Muratov, A., et al. 2009, Ap. J., 703, 1486

Quinn, P. J., Hernquist, L., \& Fullagar, D. P. 1993, Ap. J., 403, 74

Reitzel, D. B., Guhathakurta, P., \& Rich, R. M. 2004, A. J., 127, 2133

Rich, R. M., Reitzel, D. B., Guhathakurta, P., Gebhardt, K., \& Ho, L. C. 2004, A. J., 127, 2139

Richardson, J. C., Ferguson, A. M. N., Johnson, R. A., et al. 2008, A. J., 135, 1998

Richardson, J. C., Irwin, M. J., McConnachie, A. W., et al. 2011, Ap. J., 732, 76

Roberts, M. S., \& Whitehurst, R. N. 1975, ApJ., 201, 327

Sadoun, R., Mohayaee, R., \& Colin, J. 2014, MNRAS, 442, 160

Searle, L., Zinn, R. 1978, Ap. J., 225, 357

Tanaka, M., Chiba, M., Komiyama, Y., et al. 2010, Ap. J., 708, 1168

Tanvir, N. R., Mackey, A. D., Ferguson, A. M. N., et al. 2012, MNRAS, 422, 162

van der Marel, R. P., Besla, G., Cox, T. J., Sohn, S. T., \& Anderson, J. 2012, Ap. J., 753, 9

Veljanoski, J., Ferguson, A. M. N., Mackey, A. D., et al. 2013, Ap. J. Lett., 768, L33

Veljanoski, J., Mackey, A. D., Ferguson, A. M. N., et al. 2014, MNRAS, 442, 2929

Villalobos, Á., \& Helmi, A. 2008, MNRAS, 391, 1806

Walker, I. R., Mihos, J. C., \& Hernquist, L. 1996, Ap.J., 460, 121

Walterbos, R. A. M., \& Kennicutt, R. C., Jr. 1988, A\&A, 198, 61

Watkins, L. L., Evans, N. W., Belokurov, V., et al. 2009, MNRAS, 398, 1757

White, S. D. M., \& Rees, M. J. 1978, MNRAS, 183, 341

White, S. D. M., \& Frenk, C. S. 1991, Ap. J., 379, 52

Zinn, R. 1993, in Smith, G. H., Brodie, J. P., eds, ASP Conf. Ser. 48, The Globular Cluster-Galaxy

Connection. Astron. Soc. Pac., San Francisco, p. 38

Zolotov, A., Willman, B., Brooks, A. M., et al. 2009, Ap. J., 702, 1058

Zucker, D. B., Kniazev, A. Y., Bell, E. F., et al. 2004a, Ap. J. Lett., 612, L117

Zucker, D. B., Kniazev, A. Y., Bell, E. F., et al. 2004b, Ap. J. , 612, L121

Zucker, D. B., Kniazev, A. Y., Martínez-Delgado, D., et al. 2007, Ap. J. , 659, L21 


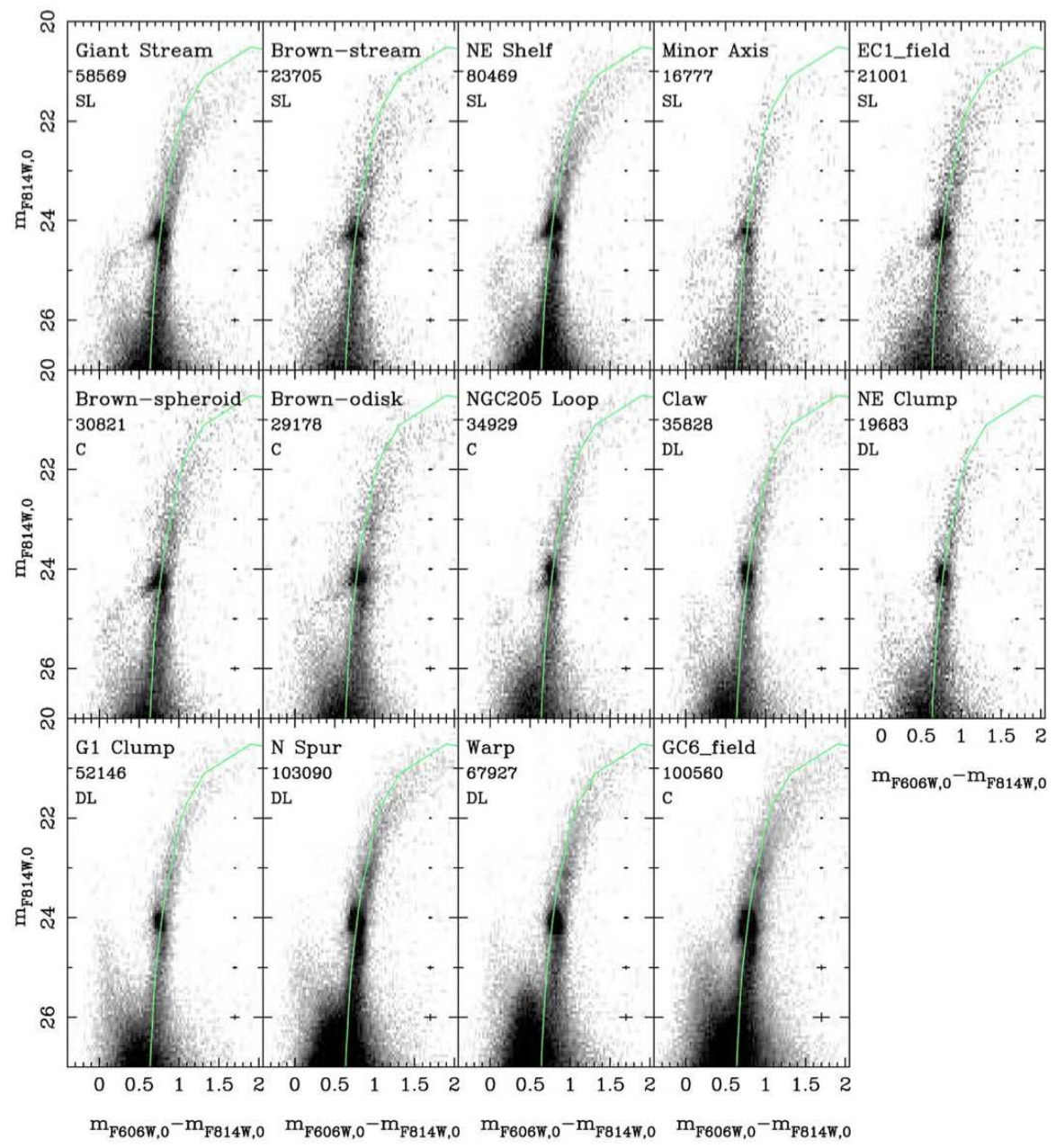

Fig. 6: HST/ACS Hess diagrams of 14 fields in the inner halo of M31, many of which lie on substructures seen in Fig. 22 The ridge line of $47 \mathrm{Tuc}([\mathrm{Fe} / \mathrm{H}]=-0.7$ and age $12.5 \mathrm{Gyr}$ ) has been shifted to the distance of M31 and overlaid in each case. Based on their CMD morphologies, the fields can be segregated into two classes; stream-like (SL) fields with tilted red clumps and extended horizontal branches and disk-like (DL) fields with round red clumps and a population of blue plume stars. Composite (C) fields have features in common with both and likely represent cases where different material is projected along the line-of-sight. Reproduced from Richardson et al. (2008). 

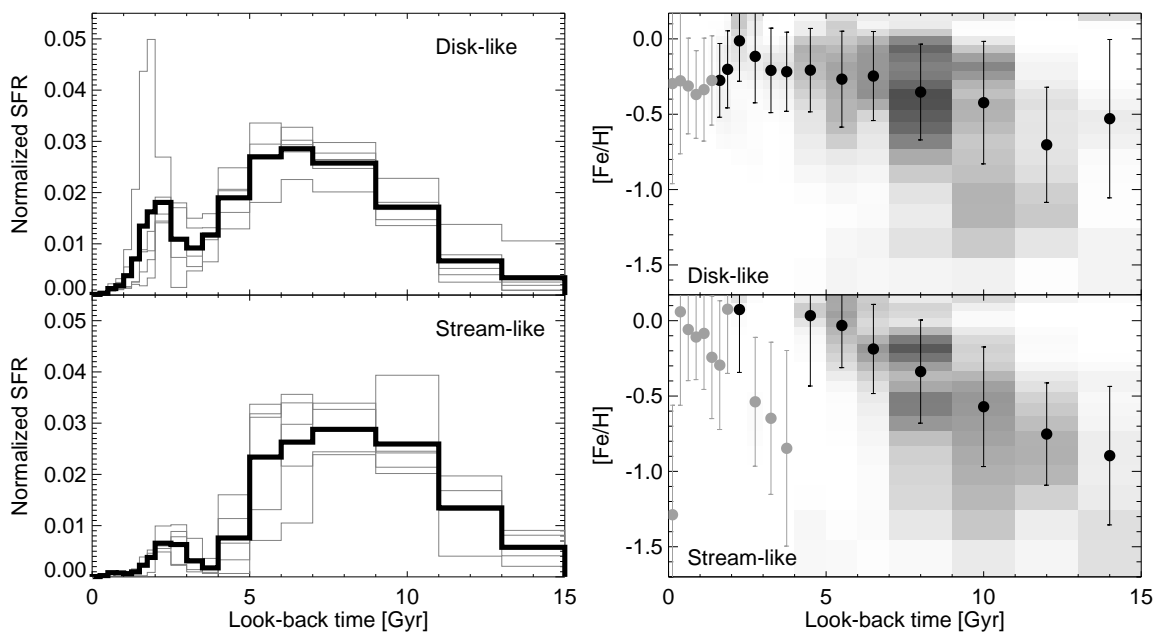

Fig. 7: (Left) The star formation histories (SFHs) and (Right) age-metallicity relations (AMRs) of M31 inner halo substructure fields, derived from quantitative fitting of the CMDs in Fig. 6 The SFHs for the individual fields are shown in light grey and are normalised to the total mass of stars formed in each field. Overlaid in bold are the average behaviours of the normalised SFHs. The filled circles in the AMRs show the median metallicity in each age bin, with grey circles indicating those bins which contain $\leq 1 \%$ of the total stellar mass and hence carry significant uncertainties. There is more star formation at early (late) times in the stream (disk) fields and the stream fields also exhibit a more rapid early chemical evolution. 


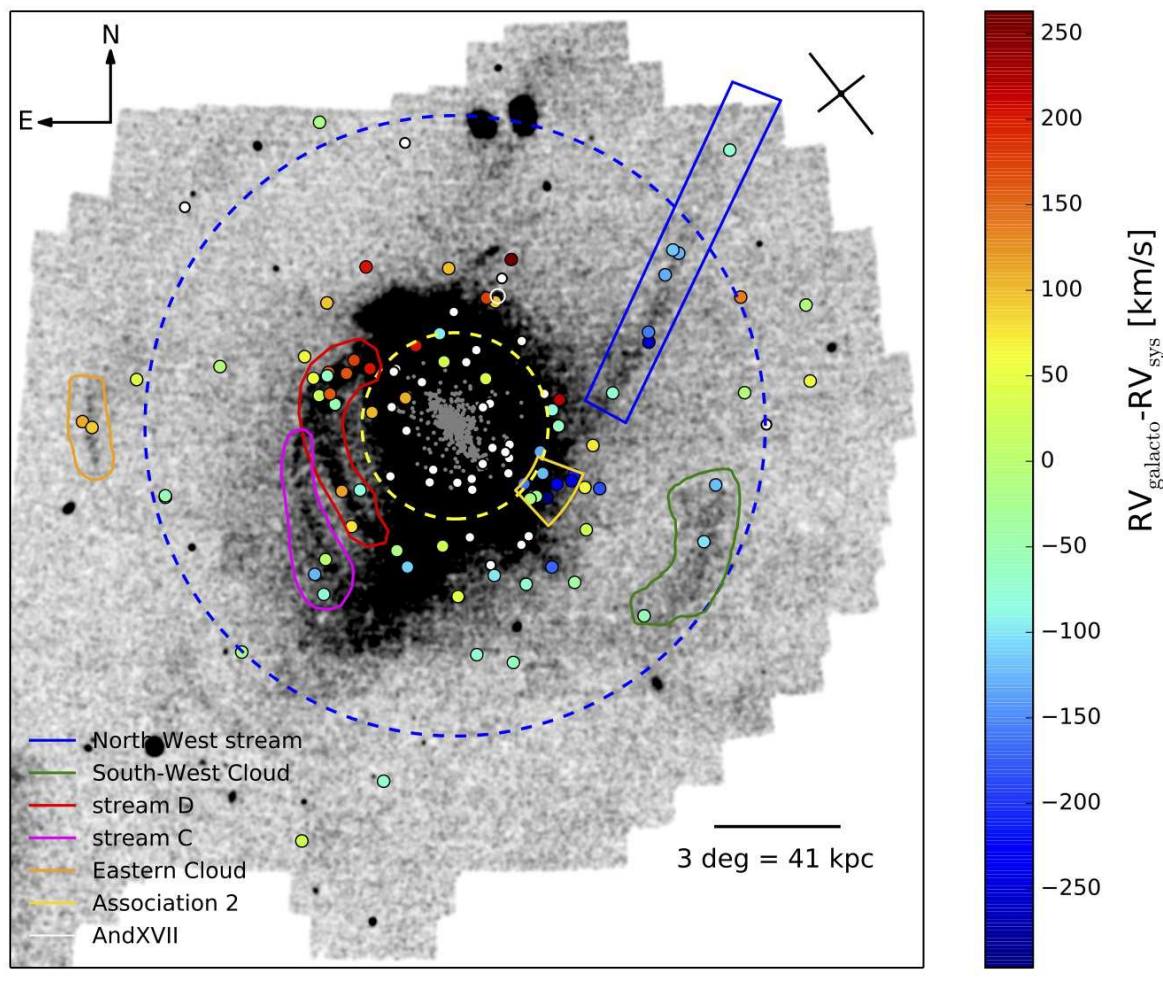

Fig. 8: Map showing extant radial velocity measurements for M31 outer halo GCs, projected on top of the PAndAS metal-poor RB map. Most of the outer halo GCs can be seen to preferentially lie along stellar streams. Inner halo GCs from the Revised Bologna Catalogue are shown as grey points. The GCs are colour-coded by their radial velocity in the M31-centric frame (white points indicate those objects with no radial velocity measurement), and the inner and outer dashed circles correspond to radii of 30 and $100 \mathrm{kpc}$. A clear rotational signature is seen with GCs in the NE side of the galaxy receding while those in the SW quadrant approach us. Additionally, coherent velocities are seen for GCs which lie along specific debris features, strongly suggesting that the GCs have been brought into M31 along with their host galaxies. 

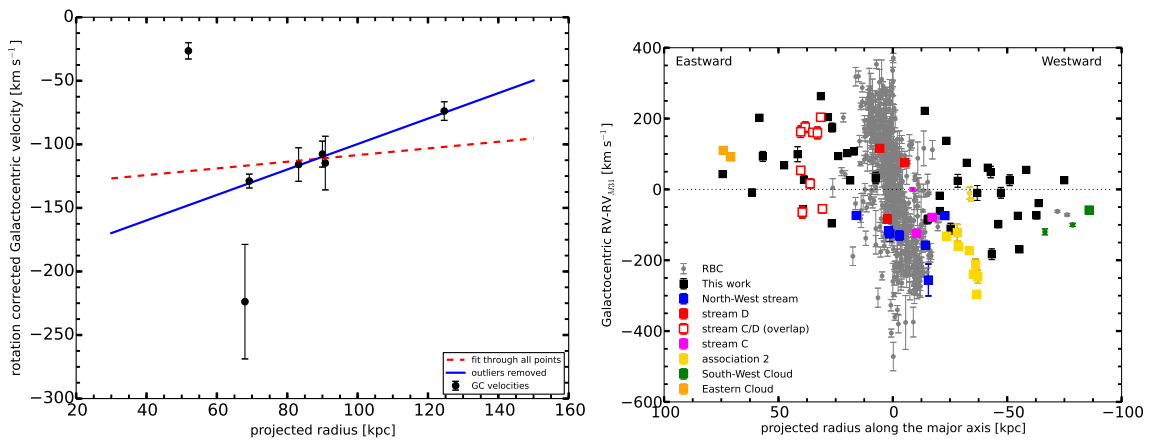

Fig. 9: (Left) Radial velocities for seven GCs that lie along the NW Stream. A clear signature of radial infall is evident. (Right) GC velocities, in the Galactocentric frame and corrected for the M31 systemic motion, versus distance along the M31 major axis. GCs which lie along specific debris features in the outer halo are colourcoded accordingly, while inner GC velocities taken from the literature are shown in light grey. The outer halo GCs rotate in the same sense as the inner GCs but with a somewhat smaller amplitude. The rotation is exhibited by the ensemble system of outer halo GCs and is not driven by specific stream features. Both panels are reproduced from Velianoski et al. (2014). 